\title{
ALGEBRAIC QUANTUM HYPERGROUPS OF DISCRETE TYPE
}

\author{
SHUANHONG WANG
}

\begin{abstract}
In this paper we will study some structures of algebraic quantum hypergroups. First, we construct more examples of algebraic quantum hypergroups of discrete type. Next, we introduce the notion of a generalized quasi-Frobenius multiplier Hopf algebra and then show that generalized quasiFrobenius multiplier Hopf algebras are a class of algebraic quantum hypergroups of discrete type. We also give some equivalent conditions for an algebraic quantum group to be of discrete type. Finally, we study sub-algebraic quantum hypergroups of discrete type and quotients of algebraic quantum hypergroups of discrete type.
\end{abstract}

\section{Introduction}

The concept of a multiplier Hopf algebra introduced by Van Daele in [4] extends the notion of a Hopf algebra to the setting of nonunital algebras. An important difference with the situation for ordinary Hopf algebras is that the comultiplication of a multiplier Hopf algebra $A$ takes values in the multiplier algebra $M(A \otimes A)$ and not in $A \otimes A$ itself. Because of the occurrence of multipliers, certain constructions for Hopf algebras need to be carried out more carefully in this context.

Algebraic quantum groups are regular multiplier Hopf algebras with integrals. They have nice properties like admitting a dual quantum group and satisfying the analogue of the Pontryagin duality [6]. We notice that the study of regular multiplier Hopf algebras of discrete type was given in 1999 by Van Daele and Zhang [7].

Recently, motivated by hypergroups, algebraic quantum hypergroups were introduced by Delvaux and Van Daele in [2]. They generalize algebraic quantum groups in the following sense. An algebraic quantum hypergroup is an associative algebra with a non-degenerate product, with a regular coproduct $\Delta: A \rightarrow M(A \otimes A)$ which is not necessarily a homomorphism of algebras, with counit $\varepsilon$, with a faithful integral $\psi$ and with an antipode $S: A \rightarrow A$ that is a bijective antimorphism of algebras satisfying: $S((\psi \otimes \iota)(b \otimes 1) \Delta(a))=$ $(\psi \otimes \iota)(\Delta(b)(a \otimes 1))$ for all $a, b \in A$. A basic example of algebraic quantum 
hypergroup is the algebra $C_{c}(G)$ of compactly supported functions on a discrete group $G$ with a finite subgroup $H$ of order $n$. The coproduct $\Delta: C_{c}(G) \rightarrow$ $M\left(C_{c}(G) \otimes C_{c}(G)\right)$ is given by $\Delta(f)(p, q)=\frac{1}{n} \sum_{h \in H} f(p h q)$ for all $p, q \in$ $G$. These algebraic quantum hypergroups were recently generalized to the setting of bornological quantum hypergroups in [6].

In this paper we will provide new examples of algebraic quantum hypergroups that are not algebraic quantum groups and study some structures of algebraic quantum hypergroups.

An outline of the paper is as follows. In Section 1, we construct more examples of algebraic quantum hypergroups of discrete type (see Example 1.5 and Example 1.8). These examples can be regarded as generalizations of a wellknown example of algebraic quantum groups given in [3, p. 496], see also [1, 2.2.1]. In Section 2, we introduce the notion of a generalized quasi-Frobenius multiplier Hopf algebra (see Definition 2.1) and then show that generalized quasi-Frobenius multiplier Hopf algebras are a class of algebraic quantum hypergroups of discrete type (see Theorem 2.9). We also give a characterization of generalized quasi-Frobenius multiplier Hopf algebras (see Theorem 2.13), generalizing the results in [7]. In Section 3, we give some equivalent conditions for an algebraic quantum group to be of discrete type (see Theorem 3.7). In Section 4, we study sub-algebraic quantum hypergroups of discrete type (see Theorem 4.1) and quotients of algebraic quantum hypergroups of dicrete type (see Theorem 4.5).

\section{More Examples of Algebraic Quantum Hypergroups}

The following definition can be found in [2] or [6].

Definition 1.1. The data $(A, m, \Delta, \varepsilon, S, \psi)$ is said to be an algebraic quantum hypergroup if the following conditions hold:

- $(A, m)$ is an associative algebra with a non-degenerate product $m$.

- $\Delta$ is a regular coproduct on $A$, i.e., $\Delta: A \rightarrow M(A \otimes A)$ is a linear map such that $\Delta(a)(1 \otimes b), \Delta(a)(b \otimes 1),(a \otimes 1) \Delta(b)$ and $(1 \otimes a) \Delta(b)$ belong to $A \otimes A$ for all $a, b \in A$ and such that

$$
(a \otimes 1 \otimes 1)(\Delta \otimes \iota)(\Delta(b)(1 \otimes c))=(\iota \otimes \Delta)((a \otimes 1) \Delta(b))(1 \otimes 1 \otimes c)
$$

for all $a, b, c \in A$.

- $\varepsilon$ is a counit on $A$, i.e., $\varepsilon: A \rightarrow k$ is a homomorphism of algebras such that $(\varepsilon \otimes \iota) \Delta(a)=a$ and $(\iota \otimes \varepsilon) \Delta(a)=a$ for all $a \in A$.

- $\psi$ is a faithful right integral on $A$, i.e., a non-zero linear functional on $A$ satisfying

$$
(\psi \otimes \iota) \Delta(a)=\psi(a) 1
$$

for all $a \in A$. 
- There is a bijective antimorphism of algebras $S: A \rightarrow A$ satisfying:

$$
S((\psi \otimes \iota)(b \otimes 1) \Delta(a))=(\psi \otimes \iota)(\Delta(b)(a \otimes 1))
$$

for all $a, b \in A$.

Let $A$ be an algebraic quantum hypergroup. An element $h \in A$ is called a right cointegral if $h a=\varepsilon(a) h$ for all $a \in A$. A similar definition for a left cointegral.

An algebraic quantum hypergroup $(A, \Delta)$ is called of discrete type if there exists a right cointegral in $A$. An algebraic quantum hypergroup $(A, \Delta)$ is called of compact type if the algebra $A$ has an identity (and hence that $\Delta(1)=$ $1 \otimes 1)$.

REMARK. (1) Observe that it is not assumed that $\Delta$ is an algebra morphism in an algebraic quantum hypergroup.

(2) ([2, Proposition 2.1]) Let $A$ be an algebraic quantum hypergroup. Then $S$ is necessarily an antimorphism of coalgebras and $\varepsilon(S(a))=\varepsilon(a)$ for all $a \in A$.

Proposition 1.2. Let A be an algebraic quantum group of discrete type with a cointegral $h \in H$. Then

$$
\Delta(h)(a \otimes 1)=\Delta(h)(1 \otimes S(a))
$$

for all $a \in A$.

Proof. We choose a right integral $\psi$ on $A$ such that $\psi(h)=1$. By Eq. (1.1), we have

$$
S(a)=(\psi \otimes \iota)(\Delta(h)(a \otimes 1)) \quad \text { for all } \quad a \in A .
$$

Hence

$$
(\psi \otimes \iota)(\Delta(h)(a b \otimes 1))=(\psi \otimes \iota)(\Delta(h)(b \otimes S(a))) \quad \text { for all } \quad a, b \in A .
$$

For all $\omega \in A^{\prime}$, by applying $\omega$ to both sides of the above equation, we get

$$
\psi(((\iota \otimes \omega) \Delta(h))(a \otimes 1) \cdot b)=\psi(((\iota \otimes \omega) \Delta(h))(1 \otimes S(a)) \cdot b)
$$

for all $a, b \in A$. Since $\psi$ is faithful, we have

$$
((\iota \otimes \omega) \Delta(h))(a \otimes 1)=((\iota \otimes \omega) \Delta(h))(1 \otimes S(a)) \quad \text { for all } \quad a \in A .
$$

Thus, we obtain

$$
\Delta(h)(a \otimes 1)=\Delta(h)(1 \otimes S(a))
$$

for all $a \in A$. This completes the proof of the proposition. 
EXAMPLE 1.3. Any regular multiplier Hopf algebra of discrete type is an algebraic quantum group and so it is an algebraic quantum hypergroup of discrete type (see [7], [2]).

ExAmPLe 1.4 (Motivating example, see [2]). Given a group $G$ with identity $e$ and a finite subgroup $H$ of $G$. Let $A$ be the algebra of complex functions with finite support on $G$ and constant on double $H$-cosets with pointwise product. The coproduct $\Delta$ is defined by

$$
\Delta(f)(p, q)=\frac{1}{n} \sum_{h \in H} f(p h q)
$$

where $n$ is order of $H$ and $p, q \in G$ and $f \in A$. The counit $\varepsilon$ is given by $\varepsilon(f)=f(e)$. The right integral $\psi$ is given by $\psi(f)=\sum_{p \in G} f(p)$ and the left integral $\varphi$ is equal to $\psi$. The right cointegral $t$ in $A$ is the function that is 1 on $H$ and 0 everywhere else. In this case, a right cointegral is also a left cointegral. By [2, Example 3.16], we know that $A$ is an algebraic quantum hypergroup of discrete type. The map $S: A \rightarrow A$ defined by $S(f)(p)=f\left(p^{-1}\right)$ satisfies the following property:

$$
\begin{aligned}
S(f)(p) & =((\psi \otimes \iota)(\Delta(t)(f \otimes 1)))(p)=\sum_{q \in G}(\Delta(t)(f \otimes 1))(q, p) \\
& =\sum_{q \in G} f(q) \Delta(t)(q, p)=\sum_{q \in G} f(q) \frac{1}{n} \sum_{h \in H} t(q h p) \\
& =\sum_{h, l \in H} \frac{1}{n} f\left(l p^{-1} h\right)=f\left(p^{-1}\right)
\end{aligned}
$$

for all $f \in A$ and $p \in G$. It is straightforward to check that the following identities in general do not hold for $A$ :

$$
\begin{aligned}
& m((S \otimes 1)(\Delta(f)(1 \otimes g)))=\varepsilon(f) g, \\
& m((1 \otimes S)((f \otimes 1) \Delta(g)))=\varepsilon(g) f
\end{aligned}
$$

for all $f, g \in A$.

We now recall that the well-known multiplier Hopf algebra $B$ in [3, p. 496] (see also $[1,2.2 .1]$ ) is a vector space with the linear basis given by $\left\{\omega_{p, i} \mid p \in\right.$ $\mathrm{Z}$ and $i=0,1\}$. The product in $B$ is given by the formulas

$$
\omega_{p, i} \omega_{q, j}=\delta_{p-q, j} \omega_{q, i+j}
$$

for all $p, q \in \mathrm{Z}$ and $i, j \in\{0,1\}$. 
The coalgebra structure on $B$ is given by the following formula. Take $p \in \mathbf{Z}$ and $i \in\{0,1\}$

$$
\Delta\left(\omega_{p, i}\right)=\sum_{r \in Z, i \geq s \in\{0,1\}}(-1)^{(p-r) s} \omega_{r, s} \otimes \omega_{p-r, i-s} .
$$

Observe that the unit in $M(B)$ is given as $1=\sum_{j \in Z} \omega_{j, 0}$.

The following example is a generalization of the above example in order to get an infinite-dimensional algebraic quantum hypergroup.

EXAmPLe 1.5. Fix a natural number $n \in \mathrm{N}$ and set $\mathrm{N}_{\leq n}=\{0,1,2, \ldots, n\}$. We define $A^{\leq n}$ as the complex infinite-dimensional algebra generated by a set $\left\{X_{i, p}, Y_{j, q} \mid p, q \in \mathrm{Z}, i, j \in \mathrm{N}_{\leq n}\right\}$ with the following relations:

$$
\begin{aligned}
& X_{i, p} X_{j, q}= \begin{cases}\delta_{p, q} X_{i+j, p}, & i+j \leq n, \\
0, & i+j>n,\end{cases} \\
& X_{i, p} Y_{j, q}= \begin{cases}\delta_{p, q+1} Y_{i+j, q}, & i+j \leq n, \\
0, & i+j>n,\end{cases} \\
& Y_{j, q} X_{i, p}= \begin{cases}\delta_{p, q} Y_{i+j, q}, & i+j \leq n, \\
0, & i+j>n,\end{cases} \\
& Y_{i, p} Y_{j, q}=0
\end{aligned}
$$

for all $p, q \in \mathrm{Z}$ and $i, j \in \mathrm{N}_{\leq n}$.

- The coproduct on $A^{\leq n}$ is given by:

$$
\begin{aligned}
\Delta\left(X_{0, p}\right)= & \sum_{r \in \mathrm{Z}} X_{0, r} \otimes X_{0, p-r}, \quad \Delta\left(X_{n, p}\right)=\sum_{r \in \mathrm{Z}} \sum_{i=0}^{n} X_{i, r} \otimes X_{n-i, p-r}, \\
\Delta\left(X_{i, p}\right)= & \sum_{r \in \mathrm{Z}} X_{0, r} \otimes X_{i, p-r}+\sum_{r \in \mathrm{Z}} X_{i, r} \otimes X_{0, p-r} \quad \text { for all } 1 \leq i<n, \\
\Delta\left(Y_{0, p}\right)= & \sum_{r \in \mathrm{Z}}(-1)^{r} X_{0, r} \otimes Y_{0, p-r}+\sum_{r \in \mathrm{Z}} Y_{0, r} \otimes X_{0, p-r}, \\
\Delta\left(Y_{n, p}\right)= & \sum_{r \in \mathrm{Z}} \sum_{i=0}^{n}(-1)^{r} X_{i, r} \otimes Y_{n-i, p-r}+\sum_{r \in \mathrm{Z}} \sum_{i=0}^{n} Y_{i, r} \otimes X_{n-i, p-r}, \\
\Delta\left(Y_{i, p}\right)= & \sum_{r \in \mathrm{Z}}(-1)^{r} X_{0, r} \otimes Y_{i, p-r}+\sum_{r \in \mathrm{Z}} Y_{0, r} \otimes X_{i, p-r} \\
& +\sum_{r \in \mathrm{Z}}(-1)^{r} X_{i, r} \otimes Y_{0, p-r}+\sum_{r \in \mathrm{Z}} Y_{i, r} \otimes X_{0, p-r} \quad \text { for all } 1 \leq i<n
\end{aligned}
$$

for all $p \in \mathbf{Z}$. 
It is straightforward to check that $\Delta$ as above is coassociative.

- The counit on $A^{\leq n}$ is given by:

$$
\varepsilon\left(X_{i, p}\right)=\delta_{i, 0} \delta_{p, 0} \quad \text { and } \quad \varepsilon\left(Y_{i, p}\right)=0
$$

for all $p \in \mathrm{Z}$ and $i \in \mathrm{N}_{\leq n}$.

If $n \geq 2$, it is straightforward to check that $\Delta$ is not an algebra map. For example, take $n=5$. We have $X_{5, p}=X_{4, p} X_{1, p}$ and

$$
\begin{aligned}
\Delta\left(X_{4, p}\right) \Delta\left(X_{1, p}\right)= & \left(\sum_{r \in \mathrm{Z}} X_{0, r} \otimes X_{4, p-r}+\sum_{r \in \mathrm{Z}} X_{4, r} \otimes X_{0, p-r}\right) \\
& \times\left(\sum_{t \in \mathrm{Z}} X_{0, t} \otimes X_{1, p-t}+\sum_{t \in \mathrm{Z}} X_{1, r} \otimes X_{0, p-t}\right) \\
= & \sum_{r \in \mathrm{Z}} X_{0, r} \otimes X_{5, p-r}+\sum_{r \in \mathrm{Z}} X_{1, r} \otimes X_{4, p-r} \\
& +\sum_{t \in \mathrm{Z}} X_{4, t} \otimes X_{1, p-t}+\sum_{t \in \mathrm{Z}} X_{5, r} \otimes X_{0, p-t}
\end{aligned}
$$

and this is not equal to $\Delta\left(X_{5, p}\right)$ for all $p \in \mathrm{Z}$.

The right cointegral in $A^{\leq n}$ is given by $Y_{n, 0}$ and the left cointegral in $A^{\leq n}$ is given by $Y_{n,-1}$. The right integral $\psi$ is given by:

$$
\psi\left(X_{i, p}\right)=0, \quad \psi\left(Y_{i, p}\right)=\delta_{i, n}
$$

for all $p \in \mathrm{Z}$ and $i \in \mathrm{N}_{\leq n}$. The left integral $\varphi$ is given by:

$$
\varphi\left(X_{i, p}\right)=0, \quad \varphi\left(Y_{i, p}\right)=\delta_{i, n}(-1)^{p}
$$

for all $p \in \mathbf{Z}$ and $i \in \mathrm{N}_{\leq n}$.

- Define a linear map $S$ on $A^{\leq n}$ by:

$$
S\left(X_{i, p}\right)=X_{i,-p}, \quad S\left(Y_{i, p}\right)=(-1)^{p+1} Y_{i,-p-1}
$$

for all $p \in \mathbf{Z}$ and $i \in \mathrm{N}_{\leq n}$. Then $S$ is bijective.

It is straightforward to verify that $S$ is both an anti-algebra map and an anticoalgebra map. It is also straightforward to check that the following identities in general do not hold for $A$ :

$$
\begin{aligned}
& m((S \otimes 1)(\Delta(a)(1 \otimes b)))=\varepsilon(a) b, \\
& m((1 \otimes S)((a \otimes 1) \Delta(b)))=\varepsilon(b) a
\end{aligned}
$$

for all $a, b \in A^{\leq n}$. 
Remark 1.6. In Example 1.5, we consider $A^{\leq 0}$, which has the linear basis given by $\left\{X_{0, p}, Y_{0, p} \mid p \in \mathrm{Z}\right\}$. Then there is the following isomorphism as multiplier Hopf algebras:

$$
\Theta: A^{\leq 0} \rightarrow B^{\text {cop }}, \quad X_{0, p} \mapsto \omega_{p, 0}, \quad Y_{0, p} \mapsto \omega_{p, 1}
$$

for all $p \in \mathbf{Z}$.

In particular, we have

Proposition 1.7. With the above notations as in Example 1.5. Then

(1) $A^{\leq 0}$ is an algebraic quantum group of discrete type.

(2) $A^{\leq 1}$ is an algebraic quantum group of discrete type.

(3) If $n \geq 2$, then $A^{\leq n}$ is an algebraic quantum hypergroup of discrete type.

EXAMPLE 1.8. Fix an odd natural number $n \in \mathrm{N}$. Let $\mathrm{N}_{\leq n}=\{0,1,2, \ldots, n\}$. Denote the set of even numbers in $\mathbf{N}_{\leq n}$ by $\mathbf{N}_{\leq n \text { (ev) }}$ and denote the set of odd numbers in $\mathrm{N}_{\leq n-1}$ by $\mathbf{N}_{\leq n \text { (od) }}$. Let $A^{\leq n \text { (od) }}$ be the algebra as in Example 1.5 with the same counit as in Example 1.5, with the same coproduct $\Delta$ on the elements $X_{0, p}, X_{n, p}, Y_{0, p}$ and $Y_{n, p}$ as in Example 1.5, for all $p \in \mathrm{Z}$, but with a different coproduct on the elements $X_{i, p}$ and $Y_{i, p}$, given by:

$$
\begin{aligned}
\Delta\left(X_{i, p}\right)= & \sum_{r \in \mathrm{Z}} X_{0, r} \otimes X_{i, p-r}+\sum_{r \in \mathrm{Z}} X_{i, r} \otimes X_{0, p-r} \quad \text { for all } i \in \mathrm{N}_{\leq n(\mathrm{od})}, \\
\Delta\left(X_{i, p}\right)= & \sum_{r \in \mathrm{Z}} X_{0, r} \otimes X_{i, p-r}+\sum_{r \in \mathrm{Z}} X_{1, r} \otimes X_{i-1, p-r} \\
& +\sum_{r \in \mathrm{Z}} X_{i-1, r} \otimes X_{1, p-r}+\sum_{r \in \mathrm{Z}} X_{i, r} \otimes X_{0, p-r} \quad \text { for all } i \in \mathrm{N}_{\leq n(\mathrm{ev})}, \\
\Delta\left(Y_{i, p}\right)= & \sum_{r \in \mathrm{Z}}(-1)^{r} X_{0, r} \otimes Y_{i, p-r}+\sum_{r \in \mathrm{Z}} Y_{0, r} \otimes X_{i, p-r} \\
& +\sum_{r \in \mathrm{Z}}(-1)^{r} X_{i, r} \otimes Y_{0, p-r}+\sum_{r \in \mathrm{Z}} Y_{i, r} \otimes X_{0, p-r} \text { for all } i \in \mathrm{N}_{\leq n(\mathrm{od})}, \\
\Delta\left(Y_{i, p}\right)= & \sum_{r \in \mathrm{Z}}(-1)^{r} X_{0, r} \otimes Y_{i, p-r}+\sum_{r \in \mathrm{Z}} Y_{0, r} \otimes X_{i, p-r} \\
& +\sum_{r \in \mathrm{Z}}(-1)^{r} X_{1, r} \otimes Y_{i-1, p-r}+\sum_{r \in \mathrm{Z}} Y_{1, r} \otimes X_{i-1, p-r} \\
& +\sum_{r \in \mathrm{Z}}(-1)^{r} X_{i-1, r} \otimes Y_{1, p-r}+\sum_{r \in \mathrm{Z}} Y_{i-1, r} \otimes X_{1, p-r} \\
& +\sum_{r \in \mathrm{Z}}(-1)^{r} X_{i, r} \otimes Y_{0, p-r}+\sum_{r \in \mathrm{Z}} Y_{i, r} \otimes X_{0, p-r} \text { for all } i \in \mathrm{N}_{\leq n(\mathrm{ev})}
\end{aligned}
$$

for all $p \in \mathbf{Z}$. 
It is straightforward to check that $A^{\leq n(\mathrm{od})}$ have the same cointegrals, integrals and antipode as in Example 1.5.

Similarly, we have

Proposition 1.9. Take the notations as in Example 1.8. Then

(1) $A^{\leq 1(\mathrm{od})}$ is an algebraic quantum group of discrete type.

(2) If $n \geq 3$ then $A^{\leq n(\mathrm{od})}$ is an algebraic quantum hypergroup of discrete type.

\section{Generalized Quasi-Frobenius Multiplier Hopf Algebras}

Let $A$ be an algebra with a non-degenerate product. We now consider the dual space $A^{\prime}$ of $A$ as a right $A$-module for the natural action of $A$ defined by $\omega a=\omega(a \cdot)$ for all $a \in A$ and $\omega \in A^{\prime}$. We denote $A^{\prime} A$ by $\widetilde{A}$.

Definition 2.1. The data $(A, m, \Delta, \varepsilon, S, h)$ is said to be a generalized quasi-Frobenius multiplier Hopf algebra if the following conditions hold:

(a) $(A, m)$ is an associative algebra with a non-degenerate product $m$.

(b) $\Delta$ is a regular coproduct on $A$ in the sense of Definition 1.1.

(c) $\varepsilon$ is a counit on $A$ in the sense of Definition 1.1.

(d) $h \in A$ such that if $(\omega \otimes \iota) \Delta(h)=0$, then $\omega=0$ for all $\omega \in A^{\prime}$ and if $(\iota \otimes \omega) \Delta(h)=0$, then $\omega=0$ for all $\omega \in A^{\prime}$.

(e) $S: A \rightarrow A$ is an anti-automorphism of coalgebras satisfying

$$
\Delta(h)(a \otimes 1)=\Delta(h)(1 \otimes S(a))
$$

for all $a \in A$.

If moreover $A$ is a $*$-algebra and $\Delta$ is a $*$-map, then we call the data $(A, m, \Delta$, $\varepsilon, S)$ a $*$-generalized quasi-Frobenius multiplier Hopf algebra.

Remark here that for a regular multiplier Hopf algebra of discrete type, the concept of quasi-Frobenius multiplier Hopf algebra (with different notion there they call it Frobenius algebra) was first appeared in [7, Theorem 3.3].

EXAMPLE 2.2. Let $(A, \Delta)$ be an algebraic quantum hypergroup with the right integral $\psi$ on $A$. Then by [2], we have the dual $\widehat{A}=\{\psi(\cdot a) \mid a \in A\}=$ $\{\psi(a \cdot) \mid a \in A\}$ to $A$ with the following properties:

- $\widehat{A}$ is an associative algebra with a non-degenerate product given by $\left(\omega_{1} \omega_{2}\right)(x)=\left(\omega_{1} \otimes \omega_{2}\right) \Delta(x)$ for all $\omega_{1}, \omega_{2} \in \widehat{A}$ and $x \in A$ (see [2, 3.2]).

- There exists a regular coproduct $\widehat{\Delta}$ on $\widehat{A}$ given by $\langle\widehat{\Delta}(\omega), x \otimes y\rangle=\omega(x y)$ for all $\omega \in \widehat{A}$ and $x, y \in A$ (see $[2,3.2])$. 
- There is a counit $\widehat{\varepsilon}$ on $\widehat{A}$ given by $\widehat{\varepsilon}(\omega)=\psi(a)$ for all $\omega=\psi(\cdot a)$ with $a \in A$ (see [2, 3.2]).

- If $A$ has an identity, then by $[2,3.3] \psi$ is a right cointegral in $\widehat{A}$. Furthermore, if $(z \otimes \imath) \widehat{\Delta}(\psi)=0$ for all $z=\widehat{\widehat{\omega}} \in A^{\prime}$, then we compute, for all $a \in A$

$$
\langle\widehat{\Delta}(\psi), z \otimes a\rangle=0 .
$$

Hence

$$
\psi(z a)=0 .
$$

Since $\psi$ is faithful, we have $\widehat{\widehat{\omega}}=z=0$.

Similarly, we have that if $(\iota \otimes z) \widehat{\Delta}(\psi)=0$, then $z=0$ for all $z=\widehat{\omega} \in A^{\prime}$.

- There exists a map $\widehat{S}: \widehat{A} \rightarrow \widehat{A}$ defined by $\widehat{S}(\omega)=\omega \circ S$ for all $\omega \in \widehat{A}$. Then by [2, Theorem 3.11], $\widehat{S}$ is an anti-automorphism of coalgebras. If $A$ has an identity, then we have, for all $a, b \in A$ and $\omega \in \widehat{A}$,

$$
\begin{aligned}
(\widehat{\Delta}(\psi)(\iota \otimes \widehat{S}(\omega)))(b \otimes a) & =(\psi \otimes \widehat{S}(\omega))((b \otimes 1) \Delta(a)) \\
& =\omega(S((\psi \otimes \iota)((b \otimes 1) \Delta(a)))) \\
& =\omega((\psi \otimes \iota)(\Delta(b)(a \otimes 1))) \\
& =(\widehat{\Delta}(\psi)(\omega \otimes 1))(b \otimes a)
\end{aligned}
$$

and so $\widehat{\Delta}(\psi)(\omega \otimes 1)=\widehat{\Delta}(\psi)(\iota \otimes \widehat{S}(\omega))$. Therefore, if $A$ is an algebraic quantum hypergroup of compact type, then $\widehat{A}$ is a generalized quasi-Frobenius multiplier Hopf algebra (see [2, 1.13]).

Proposition 2.3. Let $(A, m, \Delta, \varepsilon, S, h)$ be a generalized quasi-Frobenius multiplier Hopf algebra. Then

(1) The map $\Gamma: \widetilde{A} \rightarrow A$ defined by $\Gamma(\omega)=(\omega \otimes \imath) \Delta(h)$, is bijective.

(2) The map $\Gamma^{\prime}: \widetilde{A} \rightarrow A$ defined by $\Gamma^{\prime}(\omega)=(\iota \otimes \omega) \Delta(h)$, is bijective.

(3) The map $L: A^{\prime} \rightarrow M(A)$ defined by $L(\omega)=(\omega \otimes \imath) \Delta(h)$, is bijective.

(4) The map $L^{\prime}: A^{\prime} \rightarrow M(A)$ defined by $L^{\prime}(\omega)=(\iota \otimes \omega) \Delta(h)$, is bijective.

(5) The space of $M(A)$ coincides with the dual space $\widetilde{A}^{\prime}$ of $\widetilde{A}$.

(6) For all $a \in A$, if $\Delta(h)(1 \otimes a)=0$, then $a=0$.

Proof. (1)-(4). By Definition 2.1(d), we know that these maps $\Gamma, \Gamma^{\prime}, L$ and $L^{\prime}$ are injective. We have to prove that they are also surjective. Suppose, e.g., that $\Gamma$ is not surjective. Then it follows the proof of [7, Proposition 2.6] that we deduce a contradiction.

(5). Follows from the proof of [7, Proposition 2.9].

(6). For all $a \in A$, if $\Delta(h)(1 \otimes a)=0$, then we have $(1 \otimes \omega(\cdot a)) \Delta(h)=0$ for all $\omega \in A^{\prime}$. By Definition 2.1(d) we have that $\omega(\cdot a)=0$. Thus we have $a=0$. 
THEOREM 2.4. If ( $A, m, \Delta, \varepsilon, S, h)$ is a generalized quasi-Frobenius multiplier Hopf algebra, then there exists an integral on A.

Proof. By Proposition 2.3(3), we can choose $\psi \in A^{\prime}$ so that $(\psi \otimes \iota) \Delta(h)=$ $1 \in M(A)$. By Proposition 2.3(2), we may take $a \in A$ and we may write $a=\Gamma^{\prime}(\omega)$ with $\omega \in \widetilde{A}$. Now write $\omega=f(b \cdot)$ such that $\psi(a)=f(b)$. For any $x \in A$ we have

$$
\begin{aligned}
(\psi \otimes \iota)(\Delta(a)(1 \otimes c)) & =(\psi \otimes \iota)(\Delta((1 \otimes \omega) \Delta(h))(1 \otimes c \otimes 1)) \\
& =(\iota \otimes \iota \otimes \omega)((\iota \otimes \Delta)((\psi \otimes \iota) \Delta(h)))(1 \otimes c \otimes 1) \\
& =f(b) c=\psi(a) c .
\end{aligned}
$$

As the product in $A$ is non-degenerate, we get $(\psi \otimes \imath) \Delta(a)=\psi(a)$. Thus, $\psi$ is a right integral on $A$.

The proof of the following Proposition is essentially found in $[2,1.16]$.

Proposition 2.5. Let ( $A, m, \Delta, \varepsilon, S, h)$ be a generalized quasi-Frobenius multiplier Hopf algebra. Given elements $a_{1}, a_{2}, \ldots, a_{n} \in A$, there exist elements $e, f \in A$ such that $e a_{i}=a_{i}$ and $a_{i} f=a_{i}$ for all $i$.

Proof. Similar to [2, Proposition 1.6]. It goes as follows: We consider a set

$$
V=\left\{\left(a a_{1}, a a_{2}, \ldots, a a_{n}, a_{1} a, a_{2} a, \ldots, a_{n} a\right) \mid a \in A\right\}
$$

and consider a linear functional on $A^{2 n}$ that is zero on $V$. Then we have functionals $\omega_{i}$ and $\rho_{i}$ on $A$ for $i=1,2, \ldots, n$ such that

$$
\sum_{i=1}^{n} \omega_{i}\left(a a_{i}\right)+\sum_{i=1}^{n} \rho_{i}\left(a_{i} a\right)=0
$$

for all $a \in A$. Hence, for all $x, a \in A$ one has

$$
\begin{aligned}
x & \left(\sum_{i=1}^{n}\left(\omega_{i} \otimes \iota\right)\left(\Delta(a)\left(a_{i} \otimes 1\right)\right)+\sum_{i=1}^{n}\left(\rho_{i} \otimes \iota\right)\left(\left(a_{i} \otimes 1\right) \Delta(a)\right)\right) \\
& \left.=\sum_{i=1}^{n}\left(\omega_{i} \otimes \iota\right)\left((1 \otimes x) \Delta(a)\left(a_{i} \otimes 1\right)\right)+\sum_{i=1}^{n}\left(\rho_{i} \otimes \iota\right)\left(\left(a_{i} \otimes x\right) \Delta(a)\right)\right)=0 .
\end{aligned}
$$

Since the product in $A$ is non-degenerate, we get for all $a \in A$ that

$$
\left(\sum_{i=1}^{n}\left(\omega_{i} \otimes \iota\right)\left(\Delta(a)\left(a_{i} \otimes 1\right)\right)+\sum_{i=1}^{n}\left(\rho_{i} \otimes \iota\right)\left(\left(a_{i} \otimes 1\right) \Delta(a)\right)\right)=0 .
$$


Letting $a=h$ in the above equation, we obtain

$$
\left(\sum_{i=1}^{n}\left(\omega_{i}\left(\cdot a_{i}\right) \otimes \iota\right)\right) \Delta(h)+\left(\sum_{i=1}^{n}\left(\rho_{i}\left(a_{i} \cdot\right) \otimes \iota\right) \Delta(h)\right)=0 .
$$

That means:

$$
\left(\left(\sum_{i=1}^{n} \omega_{i}\left(\cdot a_{i}\right)+\sum_{i=1}^{n} \rho_{i}\left(a_{i} \cdot\right)\right) \otimes \iota\right) \Delta(h)=0 .
$$

By assumption, we have

$$
\sum_{i=1}^{n} \omega_{i}\left(\cdot a_{i}\right)+\sum_{i=1}^{n} \rho_{i}\left(a_{i} \cdot\right)=0 .
$$

The remaining statement is the same as one of [2, Proposition 1.6].

Proposition 2.6. Let A be a generalized quasi-Frobenius multiplier Hopf algebra. Then we have

(1) $S$ is an antimorphism of algebras.

(2) $\varepsilon S(a)=\varepsilon(a)$ for all $a \in A$ and $h$ is a right cointegral in $A$.

(3) For an integral $\psi$ such that $\psi(h)=1$, we have that $S(a)=(\psi \otimes$ ८) $(\Delta(h)(a \otimes 1))$.

(4) If $h$ and $h^{\prime}$ are two right cointegrals, there is a scalar $\lambda \in \mathrm{C}$ so that $h^{\prime}=\lambda h$.

(5) $\widetilde{A}=A^{\prime} A=\left\{\omega(a \cdot) \mid a \in A, \omega \in A^{\prime}\right\}$.

Proof. (1) For all $a, b \in A$, we compute

$$
\begin{aligned}
\Delta(h)(1 \otimes S(a b)) & =\Delta(h)(a b \otimes 1)=(\Delta(t)(a \otimes 1))(b \otimes 1) \\
& =\Delta(h)(b \otimes S(a))=\Delta(h)(1 \otimes S(a) S(b)) .
\end{aligned}
$$

By Proposition 2.3(6), we have that $S(a b)=S(b) S(a)$.

(2). Applying $(\iota \otimes \varepsilon)$ to both sides of Eq. (2.1), we have $h \varepsilon(a)=h \varepsilon(S(a))$ for all $a \in A$. Hence $\varepsilon(S(a))=\varepsilon(a)$ for all $a \in A$. Then by applying $(\iota \otimes \varepsilon)$ to the both sides of Eq. (2.1), we get $h a=h \varepsilon(a)$ for all $a \in A$. As required.

(3). Obvious.

(4). Let $h$ and $h^{\prime}$ be two right cointegrals. By Eq. (2.1), we have $\Delta(h)\left(h^{\prime} \otimes 1\right)=\Delta(h)\left(1 \otimes S\left(h^{\prime}\right)\right)=(1 \otimes \varepsilon) \Delta(h) \otimes S\left(h^{\prime}\right)=h \otimes S\left(h^{\prime}\right)$ 
By Proposition 2.5, these is $e \in A$ so that $h^{\prime} e=h^{\prime}$. Take $\omega \in A^{\prime}$ so that $(1 \otimes \omega) \Delta(h)=e$. Hence $h^{\prime}=\omega\left(S\left(h^{\prime}\right)\right) h$. We take $\lambda=\omega\left(S\left(h^{\prime}\right)\right)$ and finish the proof of Part (4).

(5). Straightforward.

Lemma 2.7. Let ( $A, m, \Delta, \varepsilon, S, h)$ be a generalized quasi-Frobenius multiplier Hopf algebra. Let $\psi$ be a right integral on $A$ and $t$ a right cointegral such that $\psi\left(S^{-1}(h)\right)=1$. Then we have the following formula:

$$
b=(1 \otimes \psi)\left((1 \otimes b)\left(\left(1 \otimes S^{-1}\right) \Delta(h)\right)\right)
$$

for all $b \in A$.

Proof. For all $b \in A$. By Eq. (2.1), we have that

$$
\left(1 \otimes S^{-1}\right)(\Delta(h)(b \otimes 1))=\left(1 \otimes S^{-1}\right)(\Delta(h)(1 \otimes S(b))) .
$$

By applying $(1 \otimes \psi)$ to both sides of the above equation and Proposition 2.6(1), we have that

$$
\left(1 \otimes \psi S^{-1}\right)(\Delta(h)(b \otimes 1))=(1 \otimes \psi)\left((1 \otimes b)\left(1 \otimes S^{-1}\right)(\Delta(h))\right) .
$$

Since $\psi \circ S^{-1}$ is a left integral on $A$, we have

$$
\psi\left(S^{-1}(h)\right) b=(1 \otimes \psi)\left((1 \otimes b)\left(\left(1 \otimes S^{-1}\right) \Delta(h)\right)\right)
$$

and so Eq. (2.2) is proven.

Proposition 2.8. Let ( $A, m, \Delta, \varepsilon, S, h)$ be a generalized quasi-Frobenius multiplier Hopf algebra with an integral $\psi$. Then the following identities are equivalent:

$$
\begin{aligned}
S((\psi \otimes \iota)((b \otimes 1) \Delta(a))) & =(\psi \otimes \iota)(\Delta(b)(a \otimes 1)) \\
S(a) & =(\psi \otimes \iota)(\Delta(h)(a \otimes 1))
\end{aligned}
$$

for all $a, b \in A$.

Proof. Eq. (2.3) $\Rightarrow$ Eq. (2.4): We can choose a right cointegral $h$ such that $\psi(h)=1$. Then by taking $b=h$ in Eq. (2.3) and by the properties of the counit $\varepsilon$, we get Eq. (2.4).

Eq. (2.4) $\Rightarrow$ Eq. (2.3): It follows from Lemma 2.7 that:

(2.5) $\Delta(b)(a \otimes 1)=(\Delta \otimes \iota \otimes \iota)\left((\iota \otimes \psi)\left((1 \otimes b)\left(\left(1 \otimes S^{-1}\right) \Delta(h)\right)\right)\right)(a \otimes 1 \otimes 1)$ 
for all $b \in A$. Since $S$ is an antimorphism of coalgebras, it follows from Eq. (2.4) that

$\left((S \otimes S) \Delta^{\mathrm{cop}}(a)\right)(1 \otimes b)=(\psi \otimes \iota \otimes \iota)(((\Delta \otimes \iota) \Delta(h))(a \otimes 1 \otimes b))$

for all $a, b \in A$. Applying $\left(\iota \otimes S^{-1}\right)$ to both sides of the above equation, we get

(2.6) $\quad(S \otimes \imath)\left(\left(1 \otimes S^{-1}(b)\right) \Delta^{\mathrm{cop}}(a)\right)$

$=(\psi \otimes \iota \otimes \iota)\left(\left(1 \otimes 1 \otimes S^{-1}(b)\right)\left(\left(\iota \otimes \iota \otimes S^{-1}\right)(((\Delta \otimes \iota) \Delta(h)))(a \otimes 1 \otimes 1)\right)\right)$

for all $a, b \in A$. Let $S^{-1}(b)=x$ with $x \in A$.

Since $S$ is bijective and by applying $(\psi \otimes \iota)$ to both sides of Eq. (2.5) and Eq. (2.6), respectively, we obtain Eq. (2.3).

We now prove the main result in this section.

THeorem 2.9. If ( $A, m, \Delta, \varepsilon, S, h)$ is a generalized quasi-Frobenius multiplier Hopf algebra, then $(A, m, \Delta, \varepsilon, S, h)$ is an algebraic quantum hypergroup of discrete type.

Proof. By Theorem 2.4, there is the right integral on $A$. Now we choose one integral $\psi$ so that $\psi(h)=1$. Applying $(\psi \otimes \iota)$ to both sides of Eq. (2.1), we get

$$
(\psi \otimes \iota)(\Delta(h)(a \otimes 1))=((\psi \otimes \iota) \Delta(h)) S(a)=\psi(h) S(a)=S(a)
$$

for all $a \in A$. By Proposition 2.8, we have that $(A, m, \Delta, \varepsilon, S, h)$ is an algebraic quantum hypergroup of discrete type.

As an immediate result of Theorem 2.9 and Example 2.2, we have

COROLlaRy 2.10. Let $(A, \Delta)$ be an algebraic quantum hypergroup of compact type. Then $(\widehat{A}, \widehat{\Delta})$ is an algebraic quantum hypergroup of discrete type.

REMARK 2.11. In a generalized quasi-Frobenius multiplier Hopf algebra ( $A, m, \Delta, \varepsilon, \psi, h$ ), because of Proposition 2.8, we are allowed to replace Eq. (2.3) by Eq. (2.4).

We now combine Proposition 2.6(5) and [2, Theorem 3.11] to arrive at;

Proposition 2.12. If $(A, m, \Delta, \varepsilon, S, t)$ is a generalized quasi-Frobenius multiplier Hopf algebra with right integral $\psi$, then $\widetilde{A}=\widehat{A}$ where $\widehat{A}$ is defined by

$$
\{\psi(a \cdot) \mid a \in A\} .
$$


Therefore $\widetilde{A}$ (with product and coproduct $\widehat{\Delta}$ dual to the coproduct, and product in A) have the following properties:

(1) $\{\psi(a \cdot) \mid a \in A\}=\{\psi(\cdot a) \mid a \in A\}=\{\phi(a \cdot) \mid a \in A\}=\{\phi(\cdot a) \mid a \in$ $A\}$ where $\phi$ is a left integral on $A$.

(2) There exists a right integral $\widehat{\hat{\psi}}$ on $\widehat{A}$ such that the map $\widehat{\Gamma}: \widehat{A} \rightarrow \widehat{\hat{A}}$ defined by $\widehat{a} \mapsto(\widehat{a} \otimes \iota) \hat{\widehat{\Delta}}(\widehat{\hat{\psi}})$ is bijective.

(3) There exists a right cointegral $\widehat{t}$ on $\widehat{A}$ such that the map $\Gamma: \widehat{\widehat{A}} \rightarrow \widehat{A}$ defined by $\widehat{\widehat{a}} \mapsto(\widehat{a} \otimes \iota) \widehat{\Delta}(\widehat{t})$ is bijective.

(4) There exists a counit $\widehat{\varepsilon}$ defined by $\widehat{\varepsilon}(\omega)=\psi(a)$ for all $\omega=\psi(a \cdot)$.

(5) $\varepsilon$ is a group-like element.

(6) The map $\widehat{S}: \widehat{A} \rightarrow \widehat{A}$ defined by $\widehat{S}(\omega)=(\widehat{\hat{\psi}} \otimes \iota)(\widehat{\Delta}(\widehat{t})(\omega \otimes 1))$ is an antimorphism of algebras and coalgebras.

Let $A$ be an algebra and $I$ be a left ideal, and $J$ be a right ideal. The right annihilator of $I$ is the set $r(I)=\{x \in A \mid a x=0$ for all $a \in I\}$. Similarly, the left annihilator of $J$ is the set $l(J)=\{x \in A \mid x a=0$ for all $a \in J\}$. We say that $A$ is quasi-Frobenius if for any left ideal $I$ and any right ideal $J$ we have $\operatorname{lr}(I)=I$ and $r l(J)=J$.

The following theorem is a generalization of [7, Theorem 3.6, Theorem 3.8] to a generalized quasi-Frobenius multiplier Hopf algebra.

THEOREM 2.13. The following statements are equivalent:

(1) $(A, m, \Delta, \varepsilon, S, t)$ is a generalized quasi-Frobenius multiplier Hopf algebra.

(2) Any proper ideal of A has a non-zero left annihilator.

(3) Any proper right ideal of A has a non-zero left annihilator.

(4) A is quasi-Frobenius.

\section{Algebraic Quantum Hypergroups of Discrete Type}

Let $(A, m, \Delta, \varepsilon, \psi)$ be an associative algebra with or without unit, but with non-degenerate product $m$ and with a coproduct $\Delta: A \rightarrow M(A \otimes A)$, here $\Delta$ is not necessarily an algebra morphism, and a counit $\varepsilon: A \rightarrow k$, and with a right integral $\psi$. By [2, Proposition 1.6], any finite elements of $A$ have common two-sided local units. Let $A^{\prime}$ denote the dual space of $A$. Then we have $A A^{\prime}=\left\{\omega(\cdot a) \mid a \in A, \omega \in A^{\prime}\right\}$ and $A^{\prime} A=\left\{\omega(a \cdot) \mid a \in A, \omega \in A^{\prime}\right\}$. We will denote these two submodules $A A^{\prime}$ and $A^{\prime} A$ of $A^{\prime}$ by $H_{l}$ and $H_{r}$, respectively. Remark here that $H_{l}$ and $H_{r}$ are also algebras for the product dual to the coproduct (see, e.g., [5]). 
We consider $\bullet: A \otimes H_{l} \rightarrow H_{l}$ and $\triangleleft: H_{r} \otimes A \rightarrow H_{r}$ defined as $a \triangleright f=$ $f(\cdot a)$ and $\omega \triangleleft a=\omega(a \cdot)$ for all $a \in A, f \in H_{l}$ and $\omega \in H_{r}$. Then for every $f \in A^{\prime}$, the induced maps $\neg: A \rightarrow H_{l}$ and $f \triangleleft: A \rightarrow H_{r}$ are morphism of left $A$-modules and of right $A$-modules, respectively.

Dually, one can take $>: A^{\prime} \otimes A \rightarrow M(A)$ and $४: A \otimes A^{\prime} \rightarrow M(A)$ defined as $f \triangleright a=(\iota \otimes f) \Delta(a)$ and $a \triangleleft f=(\omega \otimes \iota) \Delta(a)$ for all $a \in A$ and $f \in A^{\prime}$. Then for every $a \in A$, the induced maps $\triangleright a: A^{\prime} \rightarrow M(A)$ and $a$ ४: $A^{\prime} \rightarrow M(A)$ are linear maps, respectively.

Lemma 3.1. Let $(A, m, \Delta, \varepsilon)$ be an associative algebra with a non-degenerate product $m$, and with a regular coproduct $\Delta$ on $A$, and with a counit $\varepsilon$ on A. Assume that there exist an integral $\psi$ on $A$ and a cointegral $h \in A$ such that the map $S: A \rightarrow A$ defined by $S(a)=(\psi \otimes \iota)(\Delta(h)(a \otimes 1))$ is an anti-automorphism of algebras and coalgebras. Then

(1) The maps

$$
a \mapsto \psi \triangleleft a \quad \text { and } \quad a \mapsto a \triangleright \psi
$$

are bijective from $A$ to $H_{r}$ and from $A$ to $H_{l}$, respectively.

(2) The maps

$$
f \mapsto h \triangleleft f \quad \text { and } \quad f \mapsto f \triangleright h
$$

are bijective from $H_{r}$ to $A$ and from $H_{l}$ to $A$, respectively.

(3) The maps

$$
f \mapsto h \triangleleft f \quad \text { and } \quad f \mapsto f \triangleright h
$$

are bijective from $A^{\prime}$ to $M(A)$.

(4) $(A, m, \Delta, \varepsilon, S, \psi, h)$ is an algebraic quantum hypergroup of discrete type.

Proof. (1) We notice first that the map $\psi \triangleleft$ is injective since $\psi$ is faithful. To prove $\psi \triangleleft$ is surjective, let $x=\left(\iota \otimes \omega S^{-1}\right) \Delta(h)$, for all $\omega \in H_{r}$ and $a \in A$. We have

$$
\begin{aligned}
(\psi \varangle x)(a) & =\left(\psi \triangleleft\left(\left(\iota \otimes \omega S^{-1}\right) \Delta(h)\right)\right)(a) \\
& =\psi\left(\left(\left(\iota \otimes \omega S^{-1}\right) \Delta(h)\right) a\right) \\
& =\left(\psi \otimes \omega S^{-1}\right)(\Delta(h)(a \otimes 1)) \\
& =\omega S^{-1}((\psi \otimes \iota)(\Delta(h)(a \otimes 1))) \\
& =\omega\left(S^{-1}(S(a))\right)=\omega(a)
\end{aligned}
$$

and so $\psi \triangleleft$ is bijective. Similarly we have that the second map is also bijective. 
(2) By Part (1), since $\psi \psi$ is bijective, i.e., $A>\psi=H_{r}$. By assumption we have $S(a)=h \varangle(a \triangleright \psi)$. It follows that $h \triangleleft H_{r}=S(A)=A$, which means that $h \varangle$ is bijective. Similarly we get that the second map is also bijective.

(3) These maps are injective. To show that, e.g., the first map is surjective, for $x \in M(A)$ we define a linear map $\omega \in A^{\prime}$ by $\omega((\iota \otimes f) \Delta(h))=f(x)$ for $f \in H_{l}$. By the part (2), $\omega$ is well-defined and also linear. Then we have $f(x)=f((\omega \otimes \imath) \Delta(t))$. Since $H_{l}$ separates points in $A$, we get $x=(\omega \otimes$ ı) $\Delta(h)=h \varangle \omega$. Similarly we get that the second map is also bijective.

(4) By the part (3), we have that Definition 2.1(d) holds. By the assumption and the proof of Proposition 1.2, Definition 2.1(e) is true. Then $A$ is a generalized quasi-Frobenius multiplier Hopf algebra. It follows from Theorem 2.9 that $A$ is an algebraic quantum hypergroup of discrete type.

EXAMPLE 3.2. Let $A$ be a regular multiplier Hopf algebra of discrete type with antipode $S$. Assume that $\psi$ and $h$ are integral on $A$ and cointegral in $A$ such that $\psi(h)=1$, respectively. Then, for all $a, b, c \in A$

$(\psi \otimes \iota)((1 \otimes b)(\Delta(h)(a \otimes c)))$

$=(\psi \otimes \iota)\left((1 \otimes b)\left(\sum \Delta\left(h a_{(1)}\right)\left(1 \otimes \varepsilon\left(a_{(2)}\right) c\right)\right)\right)$

$=(\psi \otimes \iota)\left((1 \otimes b)\left(\sum \Delta(h)\left(a_{(1)} \otimes 1\right)\right)\left(1 \otimes m\left((\iota \otimes S)\left(\Delta\left(a_{(2)}(1 \otimes c)\right)\right)\right)\right)\right)$

$=(\psi \otimes \iota)((1 \otimes b \otimes 1)(\Delta \otimes 1 \otimes 1)((1 \otimes S)((h \otimes 1) \Delta(a))(1 \otimes c)))$

$=(\psi \otimes \iota)(((h \otimes b)((\iota \otimes S) \Delta(a)))(1 \otimes c))$

$=\psi(h) b S(a) c=b S(a) c$,

since the product on $A$ is degenerate we have $(\psi \otimes \iota)(\Delta(h)(a \otimes 1))=S(a)$. By Lemma 3.1, we have three bijections: $\psi<: A \rightarrow H_{r}, h<: H_{r} \rightarrow A$ and $h \varangle: A^{\prime} \rightarrow M(A)$.

Corollary 3.3. By [2, Proposition 2.1], Proposition 2.7 and Lemma 3.1, we have that every algebraic quantum hypergroup of discrete type satisfies Eq. (3.1), Eq. (3.2) and Eq. (3.3).

REMARK 3.4. Algebraic quantum groups of discrete type are not necessarily finite dimensional (see Example 1.4).

Proposition 3.5. Let $A$ be an associative algebra with a non-degenerate product. Then

(1) If $(A, \psi, \varepsilon)$ is an associative algebra with an algebra morphism counit $\varepsilon$, and $h \in A$ is (the unique element) such that $\psi \varangle h=\varepsilon$ and Eq. (3.1) holds, then $h$ is a right cointegral in $A$. 
(2) If $(A, \Delta, h)$ admits a coproduct and $\psi \in A^{\prime}$ is (the unique element) such that $h \triangleleft \psi=1 \in M(A)$ and $E q$. (3.3) holds, then $\psi$ is a right integral on $A$.

Proof. (1) By Eq. (3.1), for all $\omega \in H_{l}$ we write $\omega=a \triangleright \psi=\Delta(\psi)(1 \otimes$ a) for some $a \in A$. Then we have, for all $b \in A$

$$
\begin{aligned}
\omega(h b) & =\Delta(\psi)(h b \otimes a)=\psi(h b a)=(\psi \triangleleft h)(b a)=\varepsilon(b a) \\
& =\varepsilon(b) \varepsilon(a)=\varepsilon(b)(\psi \triangleleft h)(a)=\varepsilon(b) \psi(h a) \\
& =(\Delta(\psi)(1 \otimes a))(h \varepsilon(b))=\omega(h \varepsilon(b))
\end{aligned}
$$

and since $\omega$ is any element of $H_{l}$, we have $h b=\varepsilon(b) h$.

(2) Similar to Part (1).

COROLlary 3.6. Let $(A, m, \Delta, \varepsilon, S, \psi, h)$ be an algebraic quantum hypergroup of discrete type. Then

(1) We have the following equivalent identities;
(2a) $h$
$\psi=1 \in M(A)$
(2b) $\psi(h)=1$;
(2c) $\psi \triangleleft h=\varepsilon$;

We say that $(h, \psi)$ is an integral pair if one of these equivalent identities holds.

(2) Given a non-zero right cointegral $h \in A$, there exists a unique non-zero linear functional $\psi$ on A such that $(h, \psi)$ is an integral pair.

(3) Given a non-zero right integral $\psi$ on $A$, there exists a unique non-zero element $h \in A$ such that $(h, \psi)$ is an integral pair.

(4) If $(h, \psi)$ is an integral pair, then any other integral pair is of the form $\left(\lambda h, \frac{1}{\lambda} \psi\right), 0 \neq \lambda \in k$.

(5) If $(h, \psi)$ is an integral pair, then $S(a)=(\psi \otimes \iota)(\Delta(h)(a \otimes 1))$ for all $a \in A$.

THEOREM 3.7. Let $(A, m, \Delta, \varepsilon)$ be an associative algebra with a nondegenerate product $m$, with a regular coproduct $\Delta$ on $A$ and with a counit $\varepsilon$ on A. Assume that $S: A \rightarrow A$ is an anti-automorphism of algebras and coalgebras. Then the following statements are equivalent:

(1) There is $h \in A$ such that Eq. (3.3) and Eq. (2.1) hold.

(2) There is $\psi \in H_{r}$ such that Eq. (3.1) and Eq. (1.1) hold.

(3) $(A, m, \Delta, \varepsilon, S, \psi, h)$ is an algebraic quantum hypergroup of discrete type.

Proof. By Proposition 2.8, it is obvious that the part (3) implies the part (1) and the part (2). 
(1) $\Rightarrow(3)$. Since $h \triangleleft A^{\prime}=M(A)$, there is an element $\psi \in A^{\prime}$ such that $h \triangleleft \psi=1 \in M(A)$. We compute

$(\psi \otimes \iota)(\Delta(h)(a \otimes 1)) \stackrel{(2.1)}{=}(\psi \otimes \iota)(\Delta(h)(1 \otimes S(a)))=(h \triangleleft \psi) S(a)=S(a)$.

Hence, by Lemma 3.1, the part (3) holds.

(2) $\Rightarrow$ (3). Since $\psi \triangleleft A=H_{r}$, there is an element $h \in A$ such that $\psi \triangleleft h=\varepsilon \in H_{r}$. Then

$$
\begin{aligned}
(\psi \otimes \iota)(\Delta(h)(a \otimes 1)) & \stackrel{(1.1)}{=} S((\psi \otimes 1)(h \otimes 1) \Delta(a)) \\
& =(\psi \triangleleft h \otimes \iota)((1 \otimes S) \Delta(a)) \\
& =S((\varepsilon \otimes \iota) \Delta(a))=S(a) .
\end{aligned}
$$

Thus, by Lemma 3.1, the part (3) holds.

\section{Sub-algebraic Quantum Hypergroups of Discrete Type}

Let $(A, m, \Delta, \varepsilon, S, \psi, h)$ be an algebraic quantum hypergroup of discrete type. By a sub-algebraic quantum hypergroup of discrete type of $A$ we mean a subalgebra $E$ with $\Delta(E) \subseteq M(E \otimes E)$ and $S(E) \subseteq E$ such that $\left(E, m_{E}, \Delta_{E}=\right.$ $\left.\left.\Delta\right|_{E},\left.\varepsilon_{E}\right|_{E},\left.S_{E}\right|_{E}, \omega, v\right)$ is an algebraic quantum hypergroup of discrete type for some $\omega \in E^{\prime}$ and $v \in E$.

THEOREM 4.1. Let $(A, m, \Delta, \varepsilon, S, \psi, h)$ be an algebraic quantum hypergroup of discrete type. Let $E$ be a subalgebra with $\Delta(E) \subseteq M(E \otimes E)$ and $S(E) \subseteq E$. Suppose that $E$ has an map $\Xi: E \rightarrow E$ satisfying $m((\Xi \otimes$ $\iota)(\Delta(x)(1 \otimes y)))=\varepsilon(x) y$ and $m((\iota \otimes \Xi)((y \otimes 1) \Delta(x)))=\varepsilon(x) y$ for all $x, y \in E$. Then $E$ is a sub-algebraic quantum hypergroup of discrete type of $A$ if and only if the following condition holds:

$(\psi \triangleleft \otimes \iota)((m \otimes \iota)((\iota \otimes \Xi \otimes \iota)((\iota \otimes \Delta)((\iota \otimes P) \Delta(h)))))=\omega \otimes v$

where $\omega \in E^{\prime}$ and $v \in E$ are right integral and cointegral, respectively, where $P: A \rightarrow E$ is any linear projection.

Proof. $(\Rightarrow)$. Let $\left(E, m_{E}, \Delta_{E}=\left.\Delta\right|_{E},\left.\varepsilon_{E}\right|_{E},\left.S_{E}\right|_{E}, \omega, v\right)$ be an algebraic quantum hypergroup of discrete type. Then by $S=(\psi(\cdot) \otimes \imath) \Delta(h)$, we have $\left.S\right|_{E}=(\omega(\cdot) \otimes \iota) \Delta(v)$. Since $S(E) \subseteq E$, we have

$$
(\psi(\cdot) \otimes P) \Delta(h)=\left.S\right|_{E}=(\omega(\cdot) \otimes \iota) \Delta(v)
$$

where $P: A \rightarrow E$ is any linear projection. 
Thus,

$$
\begin{aligned}
& (\psi \triangleleft \otimes \iota)((m \otimes \imath)((\iota \otimes \Xi \otimes \iota)((\iota \otimes \Delta)((\iota \otimes P) \Delta(h))))) \\
& =(\omega \triangleleft \otimes \iota)((m \otimes \imath)((\iota \otimes \Xi \otimes \iota)(\Delta \otimes \iota) \Delta(v))) \\
& =(\omega \triangleleft \otimes \iota)((\varepsilon \otimes \iota) \Delta(v))) \\
& =\omega \otimes v \text {. }
\end{aligned}
$$

$(\Leftarrow)$. Suppose that any linear projection $P: A \rightarrow E$ satisfies Eq. (4.1) for a right integral $\omega \in E^{\prime}$ and a right cointegral $v \in E$. Then we compute

$$
\begin{aligned}
(\omega & \otimes \iota)(\Delta(v)(x \otimes y)) \\
& =(\psi \triangleleft \otimes \iota)((m \otimes \iota)((\iota \otimes \Xi \otimes \iota)((\iota \otimes \Delta)((\iota \otimes P) \Delta(h))))(1 \otimes x \otimes y)) \\
& =(\psi \otimes \iota)((\iota \otimes m(\Xi \otimes \iota) \Delta \otimes \iota)((\iota \otimes \Delta)((\iota \otimes P) \Delta(h))))(1 \otimes x \otimes y)) \\
& =(\psi \otimes \iota)((\iota \otimes \varepsilon \otimes \iota)(\iota \otimes \Delta)((\iota \otimes P) \Delta(h))(1 \otimes x \otimes y)) \\
& =(\psi \otimes \iota)((\iota \otimes P) \Delta(h)(1 \otimes x \otimes y)) \\
& =P((\psi \otimes \iota) \Delta(h)(x \otimes 1)) y \\
& =P(S(x)) y=S(x) y
\end{aligned}
$$

for all $x, y \in E$. Since the product on $E$ is non-degenerate one gets $(\omega \otimes$ $\iota)(\Delta(v)(x \otimes 1))=\left.S\right|_{E}(x)$ for all $x \in E$. The result now follows from Lemma 3.1.

Remark here that for any algebraic quantum group $A$, if $E$ is a subalgebra of $A$ with $\Delta(E) \subseteq M(E \otimes E)$ and $S(E) \subseteq E$, then by Theorem 4.1, $E$ is necessarily an algebraic quantum subgroup.

In order to explain the condition Eq. (4.1), we give two concrete examples as follows:

Example 4.2. Let $A$ be the algebra $A^{\leq 7}$ as defined in Example 1.5 with the same counit as in Example 1.5, and with the same coproduct $\Delta$ on the elements $X_{0, p}, X_{7, p}, Y_{0, p}, Y_{7, p}$ and $X_{i, p}, Y_{i, p}$ for all $i=1,2,4$ and $p \in \mathrm{Z}$ as in Example 1.5. Its coproduct on element $X_{i, p}, Y_{i, p}$ for all $i=3,5$ and $p \in \mathbf{Z}$ is the same as in Example 1.8. For the elements $X_{6, p}$ and $Y_{6, p}$ with $p \in Z$, we have a different coproduct structure as follows:

$$
\begin{aligned}
\Delta\left(X_{6, p}\right)= & \sum_{r \in \mathrm{Z}} X_{0, r} \otimes X_{6, p-r}+\sum_{r \in \mathrm{Z}} X_{2, r} \otimes X_{4, p-r} \\
& +\sum_{r \in \mathrm{Z}} X_{4, r} \otimes X_{2, p-r}+\sum_{r \in \mathrm{Z}} X_{6, r} \otimes X_{0, p-r},
\end{aligned}
$$




$$
\begin{aligned}
\Delta\left(Y_{6, p}\right)=\sum_{r \in \mathrm{Z}}(-1)^{r} X_{0, r} \otimes Y_{6, p-r}+\sum_{r \in \mathrm{Z}} Y_{0, r} \otimes X_{6, p-r} \\
+\sum_{r \in \mathrm{Z}}(-1)^{r} X_{2, r} \otimes Y_{4, p-r}+\sum_{r \in \mathrm{Z}} Y_{2, r} \otimes X_{4, p-r} \\
+\sum_{r \in \mathrm{Z}}(-1)^{r} X_{4, r} \otimes Y_{2, p-r}+\sum_{r \in Z} Y_{4, r} \otimes X_{2, p-r} \\
\quad+\sum_{r \in \mathrm{Z}}(-1)^{r} X_{6, r} \otimes Y_{0, p-r}+\sum_{r \in Z} Y_{6, r} \otimes X_{0, p-r}
\end{aligned}
$$

for all $p \in \mathbf{Z}$.

The right cointegral in $A^{\leq 7}$ is given by $Y_{7,0}$ and the left cointegral in $A^{\leq 7}$ is given by $Y_{7,-1}$. The right integral $\psi$ is defined by:

$$
\psi\left(X_{i, p}\right)=0, \quad \psi\left(Y_{i, p}\right)=\delta_{i, 7}
$$

for all $p \in \mathbf{Z}$ and $i \in \mathbf{N}_{\leq 7}$. The left integral $\varphi$ is defined by:

$$
\varphi\left(X_{i, p}\right)=0, \quad \varphi\left(Y_{i, p}\right)=(-1)^{p} \delta_{i, 7}
$$

for all $p \in \mathrm{Z}$ and $i \in \mathrm{N}_{\leq 7}$.

A bijective antipode on $A^{\leq 7}$ is given by:

$$
S\left(X_{i, p}\right)=X_{i,-p}, \quad S\left(Y_{i, p}\right)=(-1)^{p+1} Y_{i,-p-1}
$$

for all $p \in \mathrm{Z}$ and $i \in \mathrm{N}_{\leq 7}$.

It is straightforward to verify that $S$ is both an anti-algebra map and an anti-coalgebra map. Then $A$ is an algebraic quantum hypergroup of discrete type.

Let $E$ be a infinite dimensional subalgebra of $A=A^{\leq 7}$ generated by $X_{0, p}$, $X_{2, p}, X_{4, p}$ and $X_{6, p}$ for all $p \in Z$. It is obvious that we have $\Delta(E) \subseteq M(E \otimes E)$. Define a Hopf-type antipode $\Xi: E \rightarrow E$ by

$$
\begin{array}{ll}
\Xi\left(X_{0, p}\right)=X_{0,-p}, & \Xi\left(X_{4, p}\right)=-X_{4,-p}, \\
\Xi\left(X_{2, p}\right)=-X_{2,-p}, & \Xi\left(X_{6, p}\right)=X_{6,-p}
\end{array}
$$

for all $p \in \mathrm{Z}$. We define the linear projection $P: A^{\leq 7} \rightarrow E$ by $P\left(X_{j, p}\right)=X_{i, p}$ for all $j=0,2,4,6 ; P\left(X_{j, p}\right)=0$ for all $j=1,3,5,7 ; P\left(Y_{i, p}\right)=0$ for all $p \in \mathbf{Z}$ and $i \in \mathbf{N}_{\leq 7}$.

For the right cointegral $h=Y_{7,0}$ we have

$$
\begin{aligned}
(\iota \otimes P) \Delta(h)= & \sum_{r \in \mathrm{Z}} Y_{1, r} \otimes X_{6,-r}+\sum_{r \in \mathrm{Z}} Y_{3, r} \otimes X_{4,-r} \\
& \quad+\sum_{r \in \mathrm{Z}} Y_{5, r} \otimes X_{2,-r}+\sum_{r \in \mathrm{Z}} Y_{7, r} \otimes X_{0,-r} .
\end{aligned}
$$


Hence

$$
\begin{aligned}
& (\iota \otimes \iota \otimes \Delta)((\iota \otimes P) \Delta(h)) \\
& =\sum_{r, t \in \mathrm{Z}} Y_{1, r} \otimes X_{0, t} \otimes X_{6,-r-t}+\sum_{r, t \in \mathrm{Z}} Y_{1, r} \otimes X_{2, t} \otimes X_{4,-r-t} \\
& \quad+\sum_{r, t \in \mathrm{Z}} Y_{1, r} \otimes X_{4, t} \otimes X_{2,-r-t}+\sum_{r, t \in \mathrm{Z}} Y_{1, r} \otimes X_{6, t} \otimes X_{0,-r-t} \\
& \quad+\sum_{r, t \in \mathrm{Z}} Y_{3, r} \otimes X_{0, t} \otimes X_{4,-r-t}+\sum_{r, t \in \mathrm{Z}} Y_{3, r} \otimes X_{4, t} \otimes X_{0,-r-t} \\
& \quad+\sum_{r, t \in \mathrm{Z}} Y_{5, r} \otimes X_{0, t} \otimes X_{2,-r-t}+\sum_{r, t \in \mathrm{Z}} Y_{5, r} \otimes X_{2, t} \otimes X_{0,-r-t} \\
& \quad+\sum_{r, t \in \mathrm{Z}} Y_{7, r} \otimes X_{0, t} \otimes X_{0,-r-t} .
\end{aligned}
$$

Therefore,

$$
(m \otimes \iota)(\iota \otimes \iota \otimes \Delta)((\iota \otimes P) \Delta(h))=\sum_{r \in \mathrm{Z}} Y_{1, r} \otimes X_{6,0} .
$$

Finally, we have

$(\psi \triangleleft \otimes \iota)((m \otimes \iota)((\iota \otimes \Xi \otimes \iota)((\iota \otimes \Delta)((\iota \otimes P) \Delta(h)))))=\omega \otimes X_{6,0}$ where $\omega$ is defined on $E$ by

$$
\omega\left(X_{i, p}\right)=\delta_{i, 6}
$$

for all $p \in \mathrm{Z}$ and $i=0,2,4,6$. Obviously, $\omega$ and $X_{6,0}$ are the right integral and the right cointegral of $E$, respectively.

Thus by Theorem 4.1, $\left(E,\left.m\right|_{E},\left.\Delta\right|_{E},\left.\varepsilon\right|_{E},\left.S\right|_{E}, \omega, X_{6,0}\right)$ is an algebraic quantum hypersubgroup of discrete type of $A=A^{\leq 7}$.

EXAMPLE 4.3. Let $A^{\leq 7}$ be the algebraic quantum hypergroup of discrete type as in Example 1.5 when $n=7$. Let $E$ be a infinite dimensional subalgebra of $A^{\leq 7}$ generated by $X_{0, p}, X_{2, p}, X_{4, p}$ and $X_{6, p}$ for all $p \in \mathrm{Z}$. It is obvious that we have $\Delta(E) \subseteq M(E \otimes E)$. Define a Hopf-type antipode $\Xi: E \rightarrow E$ by

$$
\begin{array}{ll}
\Xi\left(X_{0, p}\right)=X_{0,-p}, & \Xi\left(X_{4, p}\right)=-X_{4,-p}, \\
\Xi\left(X_{2, p}\right)=-X_{2,-p}, & \Xi\left(X_{6, p}\right)=-X_{6,-p}
\end{array}
$$

for all $p \in \mathrm{Z}$. We define the linear projection $P: A^{\leq 7} \rightarrow E$ by $P\left(X_{j, p}\right)=X_{i, p}$ for all $j=0,2,4,6 ; P\left(X_{j, p}\right)=0$ for all $j=1,3,5,7 ; P\left(Y_{i, p}\right)=0$ for all $p \in \mathrm{Z}$ and $i \in \mathrm{N}_{\leq 7}$. 
For the right cointegral $h=Y_{7,0}$ we have

$$
\begin{aligned}
(\iota \otimes P) \Delta(h)=\sum_{r \in \mathrm{Z}} Y_{1, r} \otimes X_{6,-r}+\sum_{r \in \mathrm{Z}} Y_{3, r} \otimes X_{4,-r} \\
\quad+\sum_{r \in \mathrm{Z}} Y_{5, r} \otimes X_{2,-r}+\sum_{r \in \mathrm{Z}} Y_{7, r} \otimes X_{0,-r} .
\end{aligned}
$$

Hence

$(\iota \otimes \iota \otimes \Delta)((\iota \otimes P) \Delta(h))$

$$
\begin{aligned}
& =\sum_{r, t \in Z} Y_{1, r} \otimes X_{0, t} \otimes X_{6,-r-t}+\sum_{r, t \in Z} Y_{1, r} \otimes X_{6, t} \otimes X_{0,-r-t} \\
& \quad+\sum_{r, t \in Z} Y_{3, r} \otimes X_{0, t} \otimes X_{4,-r-t}+\sum_{r, t \in Z} Y_{3, r} \otimes X_{4, t} \otimes X_{0,-r-t} \\
& \quad+\sum_{r, t \in Z} Y_{5, r} \otimes X_{0, t} \otimes X_{2,-r-t}+\sum_{r, t \in Z} Y_{5, r} \otimes X_{2, t} \otimes X_{0,-r-t} \\
& \quad+\sum_{r, t \in Z} Y_{7, r} \otimes X_{0, t} \otimes X_{0,-r-t} .
\end{aligned}
$$

Therefore,

$(m \otimes \iota)(\iota \otimes \iota \otimes \Delta)((\iota \otimes P) \Delta(h))$

$=\sum_{r \in Z} Y_{1, r} \otimes X_{6,0}+\sum_{r \in Z} Y_{3, r} \otimes X_{4,0}+\sum_{r \in Z} Y_{5, r} \otimes X_{2,0}-2 \sum_{r \in Z} Y_{7, r} \otimes X_{0,0}$.

Finally, we have

$(\psi \triangleleft \otimes \iota)((m \otimes \iota)((\iota \otimes \Xi \otimes \iota)((\iota \otimes \Delta)((\iota \otimes P) \Delta(h)))))$

$$
=\omega \otimes X_{0,0}+\omega^{\prime} \otimes X_{2,0}+\omega^{\prime \prime} \otimes X_{4,0}+\omega^{\prime \prime \prime} \otimes X_{6,0}
$$

where $\omega, \omega^{\prime}, \omega^{\prime \prime}, \omega^{\prime \prime \prime}$ are defined on $E$ by, respectively:

$$
\omega\left(X_{i, p}\right)=-2 \delta_{i, 0}, \quad \omega^{\prime}\left(X_{i, p}\right)=\delta_{i, 2}, \quad \omega^{\prime \prime}\left(X_{i, p}\right)=\delta_{i, 4}, \quad \omega^{\prime \prime \prime}\left(X_{i, p}\right)=\delta_{i, 6}
$$

for all $p \in \mathrm{Z}$ and $i=0,2,4,6$. Thus Eq. (4.1) is not of the form $\omega \otimes v$. By Theorem 4.1, $E$ is not a sub-algebraic quantum hypergroup of discrete type of $A^{\leq 7}$.

In fact, since for $h=X_{6, p}$ with $p \in \mathrm{Z}$, we have

$$
h \triangleleft \omega=\sum_{r \in Z} \omega\left(X_{0, r}\right) X_{6, p-r}+\sum_{r \in Z} \omega\left(X_{6, r}\right) X_{0, p-r}
$$

for all $\omega \in E^{\prime}$. Thus we have $h \triangleleft E^{\prime} ¥ M(E)$. 
The following lemma is straightforward.

LEMMA 4.4. Let $A$ be an associative algebra with a nondegenerate product and $\Delta: A \rightarrow M(A \otimes A)$ is a coproduct of $A$ and $\varepsilon: A \rightarrow k$ is a counit on $A$. Let $I$ be an ideal of $A$ satisfying $\Delta(I) \subset M(A \otimes I)+M(I \otimes A)$ and $\varepsilon(I)=0$. Then there exists a coproduct $\bar{\Delta}: \bar{A} \rightarrow M(\bar{A} \otimes \bar{A})$ with a counit $\bar{\varepsilon}: \bar{A} \rightarrow k$, such that the diagrams commute:
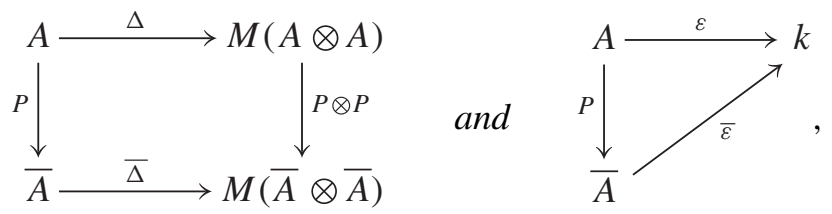

where $\bar{A}=A / I$ and $P \otimes P$ is the algebra extension in the sense of [4].

Remark here that the conditions $\Delta(I) \subset M(A \otimes I)+M(I \otimes A)$ and $\varepsilon(I)=0$ on the ideal $I$ in Lemma 4.4 means that $I$ is a 'coideal' (in the multiplier sense).

THEOREM 4.5. Let $(A, m, \Delta, \varepsilon, S, \psi, h)$ be an algebraic quantum group of discrete type. Let I be an ideal of A satisfying $\Delta(I) \subset M(A \otimes I)+M(I \otimes A)$ and $\varepsilon(I)=0$, and $S(I) \subset I$. Let $P: A \rightarrow \bar{A}=: A / I$ be the canonical projection and $\bar{S}: \bar{A} \rightarrow \bar{A}$ be the induced anti-automorphism of algebras and coalgebras. Then the following statements are equivalent:

(1) $(\bar{A}, \omega, v, \bar{S})$ is an algebraic quantum hypergroup of discrete type satisfying items (1) and (2) of Lemma 3.1.

(2) There is an element $u \in A$ such that $l(I)=A u$ and

$$
(\iota \otimes P)(\Delta(u a)(b \otimes 1))=(\iota \otimes P)((u \otimes 1) \Delta(a)(b \otimes 1))
$$

for all $a, b \in A$.

(3) There is an element $u \in A$ such that $r(I)=u A$ and

$$
(\iota \otimes P)((b \otimes 1) \Delta(a u))=(\iota \otimes P)((b \otimes 1) \Delta(a)(u \otimes 1))
$$

for all $a, b \in A$.

Proof. We only prove that the part (1) is equivalent to the part (2). Similarly, we have (1) $\Leftrightarrow$ (3). We will write $P(a)=a+I$ as $\bar{a}$ for all $a \in A$ below.

Assume that there is an element $u \in A$ such that $l(I)=A u$ and Eq. (4.2) holds. We prove that the part (1) holds. One defines the functional $\omega$ on $\bar{A}$ by $\omega(\bar{a})=\psi(u a)$. Since $u \in l(I), \omega$ is well-defined. We will apply Theorem 3.7(2) to complete the claim. 
First we only show that the map $>\omega: \bar{A} \rightarrow \bar{H}_{r}, \bar{a} \mapsto \bar{a}>\omega$ is injective. In fact, assume that $(\bar{A}>\omega)(\bar{a})=\overline{0}$. We have $(\bar{A}-\omega)(\bar{a})=\omega(\bar{a} \bar{A})=$ $\omega(\overline{a A})=\psi(u a A)$, we have $u a=0$ (since the faithfulness of $A>\psi$ ) and so $a \in r(A u)=r(l(I))=I$. Hence Eq. (3.1) holds.

Then for all $\bar{a}, \bar{b} \in \bar{A}$, we compute

$$
\begin{aligned}
(\omega \otimes \iota)(\bar{\Delta}(\bar{b})(\bar{a} \otimes 1)) & =(\omega \otimes \iota)((P \otimes P)(\Delta(b)(a \otimes 1))) \\
& =(\psi \otimes P)((u \otimes 1) \Delta(b)(a \otimes 1)) \\
& \stackrel{(4.2)}{=}(\psi \otimes \iota)((\iota \otimes P)(\Delta(u b)(a \otimes 1))) \\
& =(\iota \otimes P)((\psi \otimes \iota)(\Delta(u b)(a \otimes 1))) \\
& \stackrel{(1.1)}{=} P(S((\psi \otimes \iota)(u b \otimes 1) \Delta(a))) \\
& =P S(P((\psi \otimes \iota)(u b \otimes 1) \Delta(a))) \\
& =\bar{S}((\omega \otimes \iota)(P \otimes P)((b \otimes 1) \Delta(a))) \\
& =\bar{S}((\omega \otimes \iota)((\bar{b} \otimes 1) \bar{\Delta}(\bar{a}))) .
\end{aligned}
$$

Conversely, since $(A, \psi)$ is an algebraic quantum hypergroup of discrete type satisfying Eq. (3.1), we have $\psi \triangleleft A=H_{r}$ and so there is $u$ such that $\psi \varangle u=\omega \circ P$, i.e., $\psi(u x)=\omega(\bar{x})$ for all $x \in A$. We claim that the $u$ is a required one. In fact, since $\psi(u I A)=(\psi \varangle u)(I)=\omega(P(I))=0$ and the faithfulness of $\psi$, we have $u I=0$. This means that $u \in l(I)$, i.e., $A u \subseteq l(I)$. We also claim: $l(I) \subseteq A u$. For all $x \in r(A u)$, we have $u x=0$ and $\omega(\bar{x} \bar{A})=\omega(\overline{x A})=(\omega \circ P)(x A)=(\psi \varangle u)(x A)=\psi(u x A)=0$. Thus $\bar{x}=0$ and so $r(A u) \subseteq I$. Therefore, $l(I) \subseteq l(r(A u))=A u$ and so $l(I)=A u$.

Then we have

$$
\begin{aligned}
(\psi \otimes P)(\Delta(u a)(b \otimes 1)) & \stackrel{(1.1)}{=} \quad P(S((\psi \otimes \iota)((u a \otimes 1) \Delta(b)))) \\
= & \bar{S}((\omega \otimes \iota)((P \otimes P)((a \otimes 1) \Delta(b)))) \\
& =\bar{S}((\omega \otimes \iota)((\bar{a} \otimes 1) \bar{\Delta}(\bar{b})))) \\
\stackrel{(1.1) \text { for }}{=} \bar{A}(\omega \otimes \iota)(\bar{\Delta}(\bar{a})(\bar{b} \otimes 1)) & (\omega \otimes \iota)((P \leq P)(\Delta(a)(b \otimes 1))) \\
= & (\psi \otimes P)((u \otimes 1)(\Delta(a)(b \otimes 1))) \\
= &
\end{aligned}
$$

and so Eq. (4.2) holds since $A \triangleright \psi=H_{r}$ and $\bar{S}$ is bijective.

This finishes the proof of the theorem.

AcKnowledgement. The author would like to thank the referee for the 
helpful comments. This work was finished when the author visited Leuven under the K. U. Leuven's fellowship during March-May in 2009. He would like to thank Prof. Van Daele and his research group for warm hospitality. The work was partially supported by the NNSF of China (No. 10871042) and the NSF of Jiangsu Province (No. BK2009258). Finally, the author thanks Tianshui Ma for his help on editing the final tex-file.

\section{REFERENCES}

1. Delvaux, L., Van Daele, A., The Drinfeld' double versus the Heisenberg double for an algebraic quantum group, J. Pure Appl. Algebra 190 (2004), 59-84.

2. Delvaux, L., Van Daele, A., Algebraic quantum hypergroups, Adv. Math. 226 (2011), 11341167.

3. Delvaux, L., Van Daele, A., Wang, S. H., Quasitriangular (G-cograded) multiplier Hopf algebras, J. Algebra 289 (2005), 484-514.

4. Van Daele, A., Multiplier Hopf algebras, Trans. Amer. Math. Soc. 342 (1994), 917-932.

5. Van Daele, A., An algebraic framework for group duality, Adv. Math. 140 (1998), 323-366.

6. Van Daele, A., Wang, S. H., Pontryagin duality for bornological quantum hypergroups, Manuscripta Math. 131 (2010), 247-263.

7. Van Daele, A., Zhang, Y., Multiplier Hopf algebras of discrete type, J. Algebra 214 (1999), $400-417$.

DEPARTMENT OF MATHEMATICS

SOUTHEAST UNIVERSITY

NANJING, 210096

P. R. OF CHINA

E-mail: shuanhwang2002@yahoo.com 